\title{
In Vitro Interactions of Diclofenac with Several Types of Food
}

\author{
Fülöp Ibolya, Croitoru Mircea Dumitru*, Vajda Andrea, Jakab Irén, Fogarasi Erzsébet
}

University of Medicine and Pharmacy of Tirgu Mures, Romania

\begin{abstract}
Objective: The aim of this study was to evaluate how the membrane transport of diclofenac on presence of different types of food is modified. Methods: The interaction of diclofenac, as a pure substance and solid dispersions of the active ingredient, with different types of foods was investigated in vitro condition using a modified Franz diffusion cell. Results: The amount of diclofenac transported through a lipophilic membrane was reduced by the presence of foodstuffs in artificial gastric juice and intestinal juice also, both in the case of the pure substance and solid dispersions also. The only exception was the case of milk in artificial gastric juice, when the amount of diclofenac in the receiving compartment increased about 2-fold compared to the fasted conditions simulating media. In the case of the solid dispersions of diclofenac the membrane transport increased in all cases compared to the pure substance, but was also reduced by the foods. Conclusions: It was concluded that, the presence of different foodstuffs can influence the membrane transport of diclofenac - by inhibiting its solubility - and these differences observed in vitro can lead to modifications in the in vivo pharmacokinetic parameters. The lowest difference in diclofenac membrane transport was observed in the case of diclofenac:PEG 6000 solid dispersion prepared in 1:5 mass ratio.
\end{abstract}

Keywords: food-drug interaction, diclofenac, in vitro, membrane transport

Received: 06 June 2016 / Accepted: 11 July 2016

\section{Introduction}

Interactions between medicines and foods are widely studied. Taking the drug with food, or after a meal can influence some pharmacokinetic parameters (rate and extent of absorption, bioavailability, metabolism, etc.) of the drug $[1,2]$. The EMA (European Medicines Agency) guideline regarding the investigation of drug interactions [3] recommend to be studied, in the third phase of drug development, the effect of food intake on pharmacokinetics of the investigational drug, in order to find out the timing of medicine administration in relation to food. This has importance in reducing the inter- and intra-individual variability of pharmacokinetics of the drug. According to the EMA guideline in the food-drug interaction investigations in vivo, two types of standard meal are recommended: high-fat and moderate meal [3]. The aim of our work was to study in vitro condition the effect of different types of foodstuff on diclofenac pure substance membrane transport and to find a formulation, in which case the membrane transport of diclofenac is less influenced by the presence of food. As model drug, diclofenac (D) was chosen, because its gastrointestinal adverse effect; according to product information, it should be teak with or after meal.

Diclofenac sodium is a widely used non-steroidal anti-inflammatory drug, for treating a variety of pain and inflammatory conditions. Diclofenac can cause gastro-intestinal adverse effects by inhibiting the cyclooxygenase-1 $(\mathrm{COX}-1)$ enzyme $[4,5]$. Its pharmacological activity may be attributed mainly to inhibition of COX-2 enzyme, thus reducing the prostaglandin synthesis.

* Correspondence to: Mircea Dumitru Croitoru

E-mail: croitoru.mircea@umftgm.ro
Pharmacokinetics of diclofenac: following oral administration it is rapidly (time to maximal plasma concentration, $\mathrm{t}_{\max }$ is about $0.5-1.8 \mathrm{~h}$ ) and completely absorbed from gastro-intestinal tract, but the bioavailability is $50-60 \%$ due to its first-pass metabolism [6,7]. There are many oral formulations of diclofenac (immediate release, entericcoated tablets, etc.). Some pharmacokinetic parameters vary according to the formulation: the rate of absorption, thus $t_{\max }$ and peak plasma concentration $\left(\mathrm{C}_{\max }\right)$ are modified, while the area under plasma concentration-time curve (AUC) is not influenced [8]. The same pharmacokinetic parameters are modified by food administration in the case of pure diclofenac [9] or different diclofenac-formulations (enteric-coated tablets) [10].

\section{Materials and methods}

Sodium diclofenac was purchased from Mircrosin (Bucharest). PEG 6000, gradient grade acetonitril, $\mathrm{Na}_{2} \mathrm{HPO}_{4}$, concentrated $\mathrm{H}_{3} \mathrm{PO}_{4}$ were supplied from Merck KGaA, Germany. All other reagents and chemical substances were of analytical grade. The different types of foodstuffs were purchased from local market.

\section{Determination of membrane transport of diclofenac in the presence of different types of foodstuff}

For measurement of diclofenac transport trough membranes, a modified Franz-type diffusion cell chamber (Hanson Research Co., USA) was used. The receptor compartment was filled with $12 \mathrm{ml}$ phosphate buffer solution, $\mathrm{pH}=7.4$. The donor and receiving compartment was separated by a cellulose membrane (Teknokroma Analítika SA, Spain) with $0.45 \mu \mathrm{m}$ pore size lipophylized with sunflower 
oil. The surface of diffusion area was $1.76 \mathrm{~cm}^{2}$. The donor compartment consisted in a $30 \mathrm{ml}$ plastic chamber.

An amount of $5.6 \mathrm{mg}$ diclofenac was put in the donor compartment containing different types of foodstuffs dispersed in artificial gastric juice with pepsin $(\mathrm{pH}=1.2)$ or artificial intestinal juice with trypsin $(\mathrm{pH}=6.1)$. The studied foodstuffs were: milk $(7.5 \mathrm{ml})$, orange juice $(4.5 \mathrm{ml})$, banana $(6.3 \mathrm{~g})$, potato $(10 \mathrm{~g})$, muesli $(3 \mathrm{~g})$, salami $(1.6 \mathrm{~g})$ and standardized high-fat meal (1.6 g; mixt; containing eggs, toast with butter, bacon, hash brown potatoes and milk) according to the standard procedures for food interaction studies by EMA [3].The receiving solution was stirred by a magnetic stirrer; the donor solution was stirred by a rotating paddle. $200 \mu \mathrm{l}$ samples from the receiving chamber were withdrawn periodically (after 5, 20, $30 \mathrm{~min} ; 1,1.5,3$, 4,6 and $8 \mathrm{~h}$ ) and replaced with fresh solution.

Different mathematical models, available in the Origin software, were used for evaluation of the mechanism of drug transport, and the coefficient of determination was used for choosing the best fit.

\section{Determination of membrane transport of solid disper- sion of diclofenac in the presence of different types of food stuff}

Solid dispersions were made in 1:5 and 1:10 diclofenac:PEG 6000 mass ratio using the melting method (D 1:5; D 1:10). PEG 6000 was melted at $60-70{ }^{\circ} \mathrm{C}$ in a porcelain capsule and diclofenac was dissolved in the melted polymer; after than the mixture was kept for $24 \mathrm{~h}$ at $-20^{\circ} \mathrm{C}$. Before using it, the mixture was passed through a $355 \mu \mathrm{m}$ sieve. The membrane-transport experiments were made using the same method as described before.

\section{Quantitative determination of diclofenac in the receiv- ing compartment}

The amount of diclofenac in the receiving chamber, permeated trough the membrane, was measured using an HPLC method (Merck HPLC system: quaternary pump L-7100, auto sampler L-7200, column thermostat L-7360, DAD detector L-7455, interface L-7000, solvent degasser L-7612, HSM manager software). The measurements were carried out using a $\mathrm{pH}=2.5$ phosphate buffer:ACN (80:20) as mobile phase at $1 \mathrm{ml} / \mathrm{min}$ flow rate and a Lichrocart (250-4) Lichrospher RP-select B (5 $\mu \mathrm{m})$ column as stationary phase with detection wavelength of $276 \mathrm{~nm}$. The injection volume was $100 \mu$ l. Calculations were performed by the measurement of peak areas.

\section{Results}

Figure 1 and 2 show the amount of diclofenac transported through the lipophilic membrane in the presence of the different foodstuff.

In artificial gastric juice the in the presence of milk, the amount if diclofenac transported through the lipophilic membrane increases about 2-fold. In the other cases, the presence of food in the donor compartment inhibited the drug-transport. The least amount of diclofenac in the receiving media was observed in the presence of potato, standard of high-fat meal and banana. Based on these data, the difference between the transported diclofenac, depending on different foodstuffs, could be about 50 -fold.

From the artificial intestinal juice, in all cases, the diclofenac was transported in the receiving compartment in a less extent than in the case of the fasted conditions simulating media (blank), descending order being: orange juice,

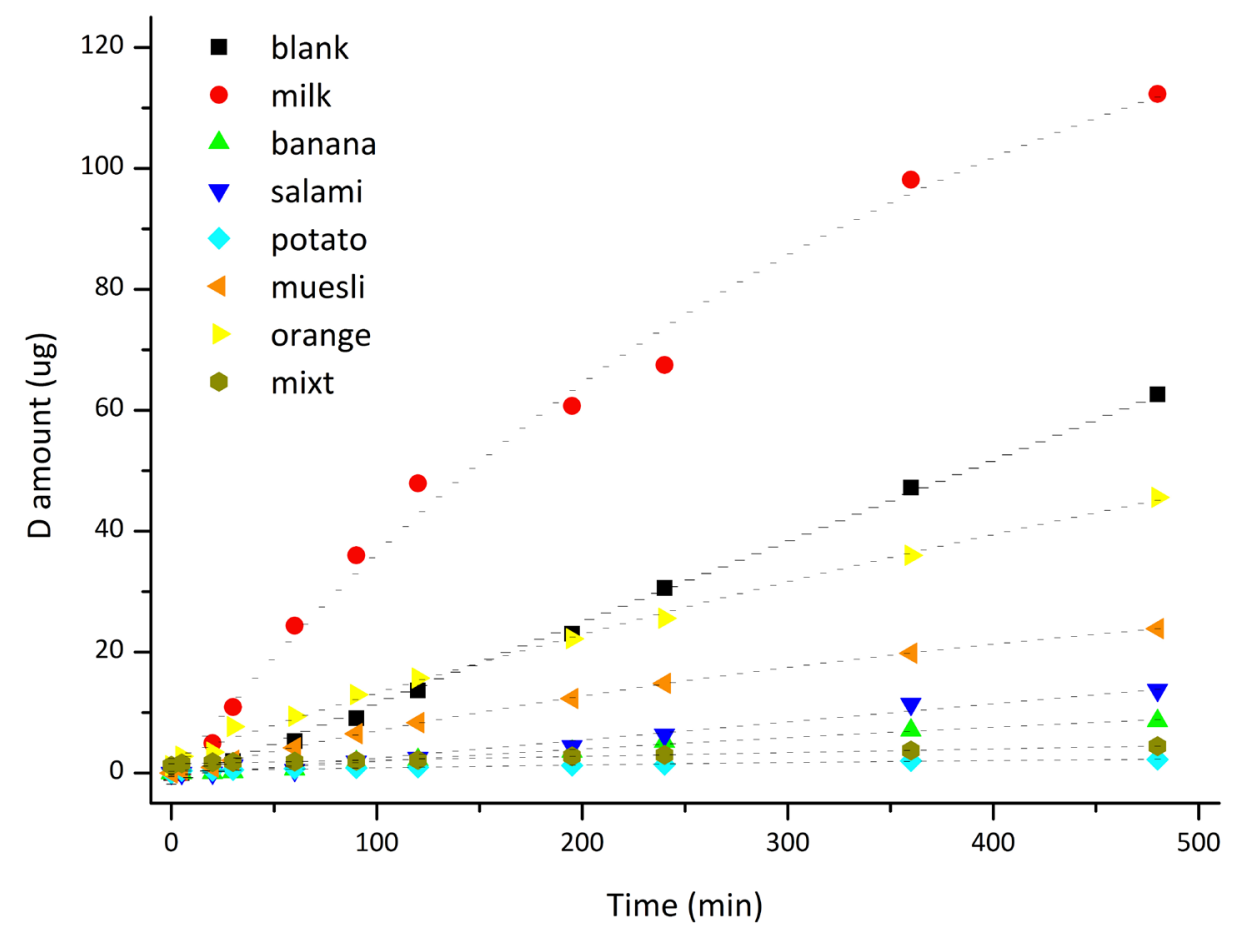

Fig. 1. Tthe amount of diclofenac transported from artificial gastric juice in the presence of the studied foodstuff Abbreviations: diclofenac-D; standardized high-fat meal - mixt 


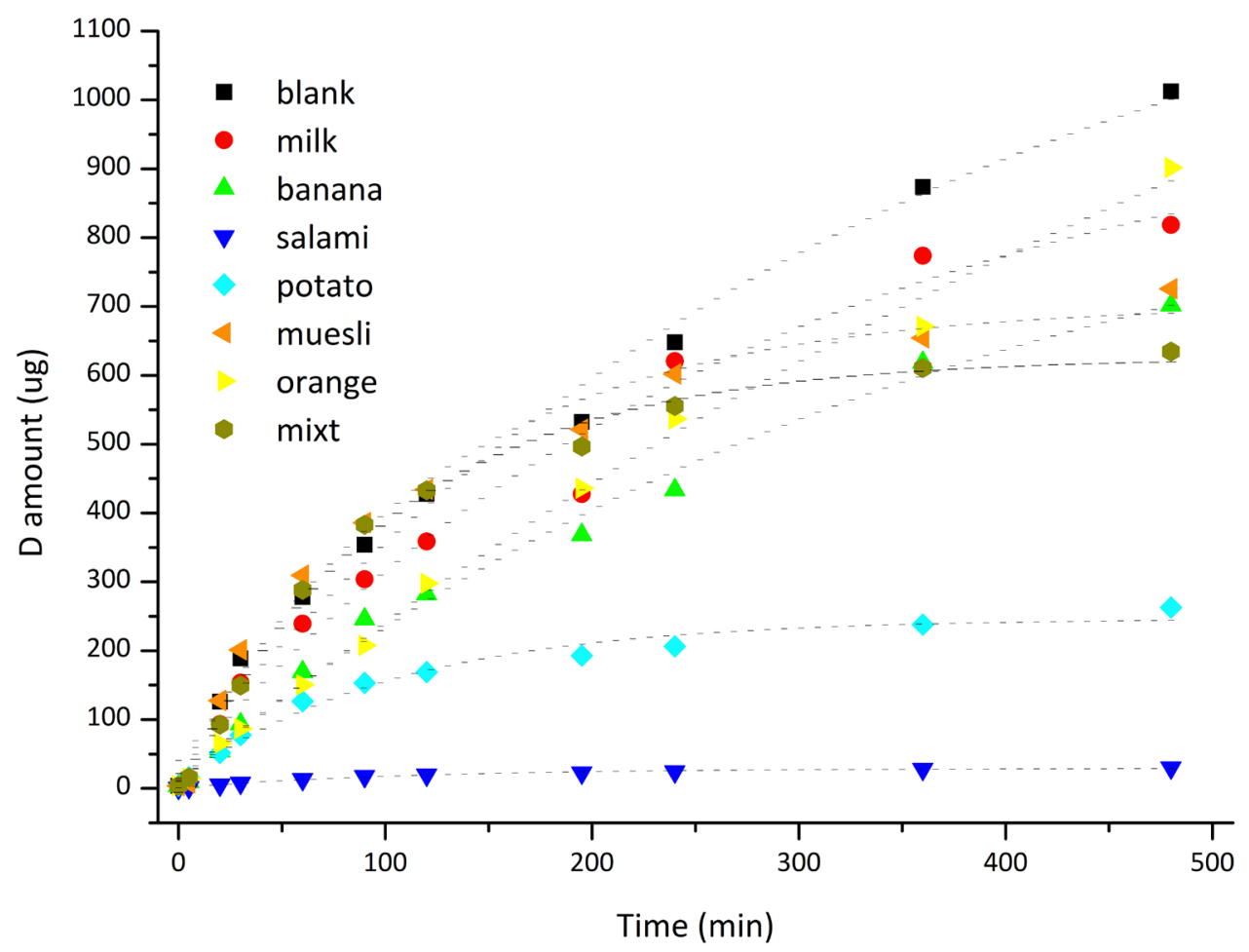

Fig. 2. The amount of diclofenac transported from artificial gastric juice in the presence of the studied foodstuffs Abbreviations: diclofenac - D; standardized high-fat meal - mixt

milk, muesli, banana, high-fat meal standard, potato and salami.

In order to verify if the membrane transport of diclofenac vary using solid dispersions of diclofenac (with an increased water-solubility and membrane transport, figure 3), instead of diclofenac pure substance, the same set of experiments were repeated with the D 1:5 and D 1:10 products. Some typical results obtained in artificial gastric juice are presented in Figure 4.

In the case of solid dispersions, the solubility, thus the membrane transport of diclofenac is increased compared to the pure substance in both dissolution media. With

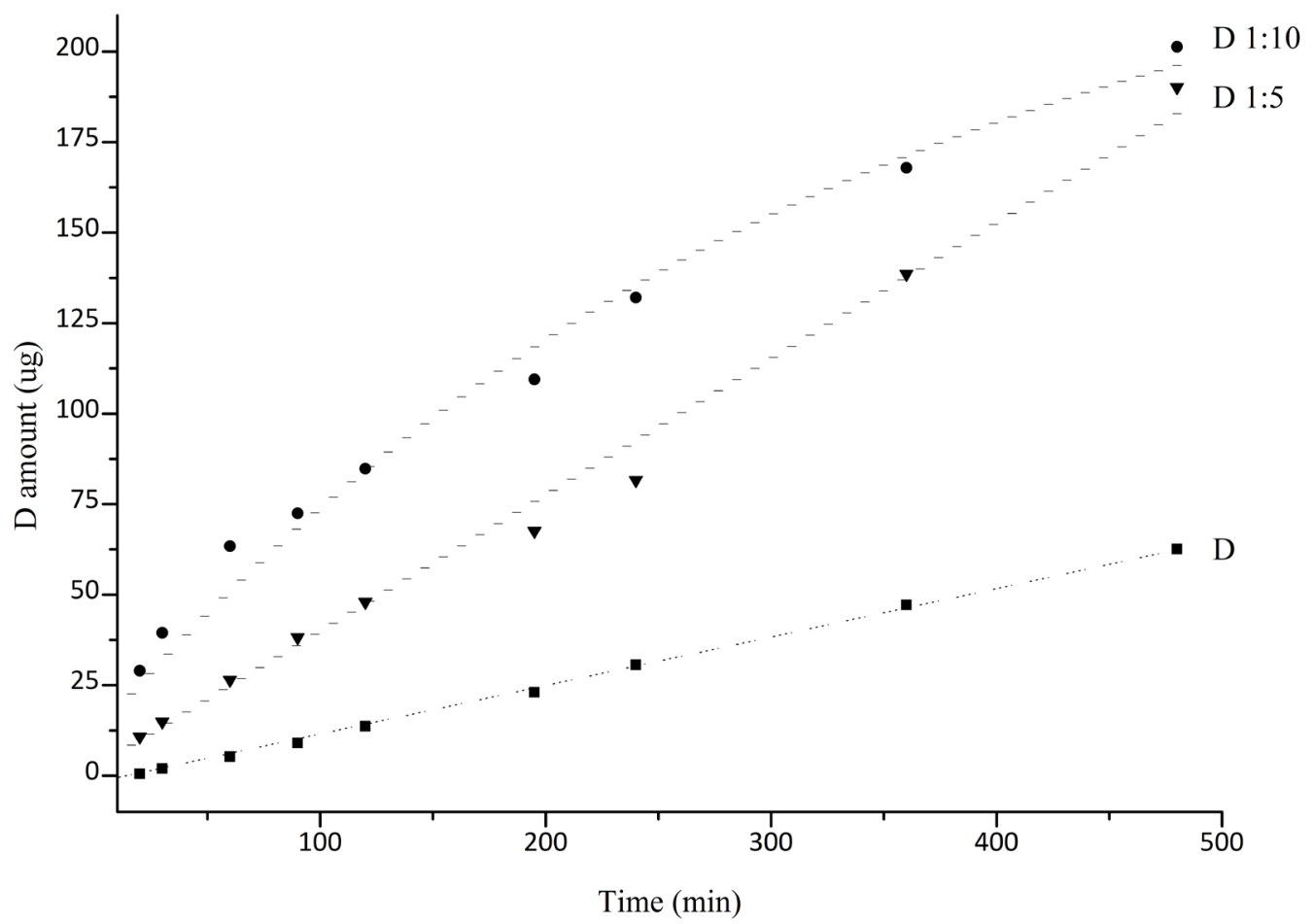

Fig. 3. Membrane transport of diclofenac and the solid dispersions in artificial gastric juice Abbreviations: diclofenac - D; solid dispersion diclofenac: PEG 6000 1:5 mass ratio - D 1:5; solid dispersion diclofenac: PEG 6000 1:5 mass ratio - D 1:10 

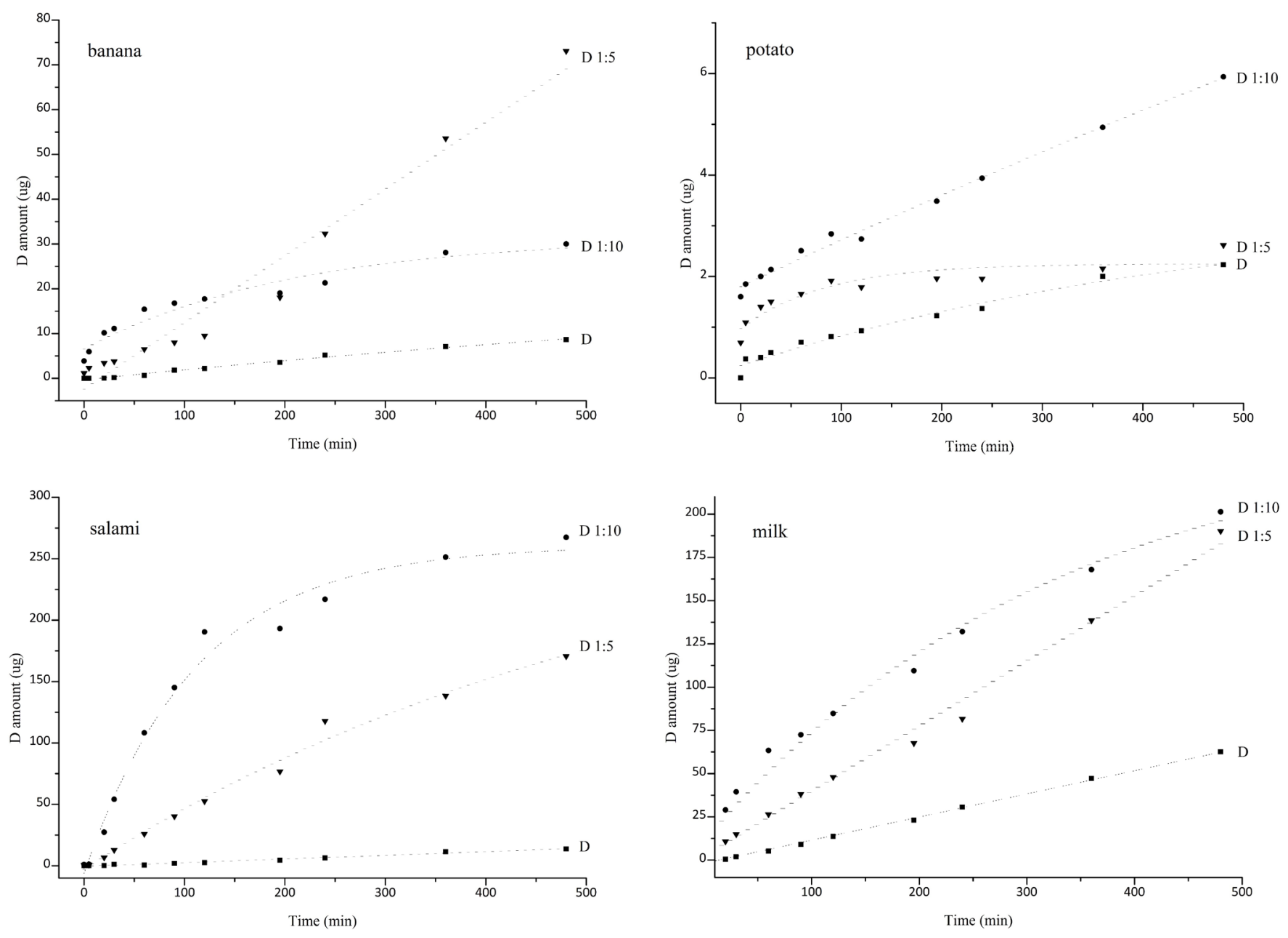

Fig. 4. Membrane transport of diclofenac from the solid dispersions in the presence of different foodstuff in artificial gastric juice Abbreviations: diclofenac - D; solid dispersion diclofenac: PEG 6000 1:5 mass ratio - D 1:5; solid dispersion diclofenac: PEG 6000 1:5 mass ratio - D 1:10

one exception (in the case of banana) the $\mathrm{D}$ 1:10 product showed the best results, regarding the amount of diclofenac transported through membrane.

The mathematical analysis of the diclofenac amount in the receiver compartment vs. time curves, shows that the mechanism of drug transport can be described by the Exponential/MnMolecular1 function ( $\left.\mathrm{y}=\mathrm{A}_{1}-\mathrm{A}_{2} \mathrm{e}^{-\mathrm{k} \cdot \mathrm{time}}\right)$, where $y$ is the amount of diclofenac in the receiving compartment, $A_{1}$ corresponds to the maximum achievable concentration, $A_{2}$ corresponds to the concentration of diclofenac in the donor compartment and $k$ is the rate constant of the transport process, expressed as the rate of concentrationdecrease in the donor compartment.

Based on the parameters obtained by the mathematical analysis of the diclofenac amount-time-curves the rate of the drug transport was the highest in the case of milk and muesli $\left(200^{*} 10^{-5} \mathrm{~min}^{-1}\right)$ in artificial gastric juice and the lowest for the salami $\left(0,03^{*} 10^{-5} \mathrm{~min}^{-1}\right)$ and blank $\left(0.07^{*} 10^{-5} \mathrm{~min}^{-1}\right)$. In the case of the solid dispersions, the $k$ parameter was almost equal or higher than those observed for diclofenac pure substance, the values were situated between $\left(37^{*} 10^{-5}\right.$ and $\left.1190^{*} 10^{-5} \mathrm{~min}^{-1}\right)$. In artificial intestinal juice the difference between the rate constants was not significant.

\section{Discussion}

In the presence of different types of foodstuffs - in almost all cases - the diclofenac membrane-transport was reduced. The only exception is the case of milk in artificial gastric juice, when the solubility, therefore the membrane transport of diclofenac was significantly increased. This can be explained by the fact, that milk reduced the acidity of artificial gastric media and the drug has higher solubility in milk digested with pepsin, compared to artificial gastric juice $[11,12]$. In the other cases, the reduced diclofenac amount in the receiving media is due to the modified viscosity of the dissolution media or binding the active ingredient to food components (e.g. fiber). The premise, that using a product (D 1:5 or 1:10) with higher solubility, can cause a reduction in the differences observed between the diclofenac solubility in the presence of the studied foodstuffs in the case of the diclofenac pure substance, was tested. Based on the observed results, the differences were reduced; the coefficient of variations between differ- 
ent type of foods (CV\%) in the receiving media decreased from $117.7 \%$ to $69.3 \%$ and $86.2 \%$ in the case of D $1: 5$ and D 1:10, respectively. Regarding the theoretically calculated rate constants the $\mathrm{CV} \%$ value was also reduce from $104.4 \%$ to $38.8 \%$ and $63.0 \%$ in the case of D $1: 5$ and D $1: 10$, respectively.

In artificial intestinal juice, the membrane-transport improvement (not shown) is not so remarkable, because the pure substance itself has a good solubility in basic media; therefore using solid dispersions instead of the pure drug did not influence significantly the membrane transport of diclofenac.

\section{Conclusion}

This study demonstrates the importance of interactions at pharmacokinetic level between different foodstuffs and drugs. The presence of food in the gastrointestinal tract can influence the dissolution and solubility thus the amount of medicine transported through membrane. This effect can induce inter- and intra-individual variability in some pharmacokinetic parameters, mainly when the drug is administered with meal, juices or milk. The aim of this study was to evaluate, using a diclofenac content solid dispersion - with higher water solubility - instead of diclofenac pure substance how the membrane transport of diclofenac in presence of different foodstuffs is modified. It was observed, that the solid dispersion of diclofenac, made in 1:5 mass ratio, is the most suitable to reduce the differences in membrane transport caused by the presence of foods in gastric juice.

\section{Acknowledgement}

The research was supported by the University of Medicine and Pharmacy of Tîrgu Mureș, internal grant number 3/23.12.2014.

\section{Conflicts of interest}

The authors report no conflicts of interest.

\section{References}

1. Yaheya $M$, Ismail $M$ - Drug-food interactions and role of pharmacist. Asian J Pharm Clin Res. 2009;2:1-10.

2. Otles S, Senturk A - Food and Drug Interactions: A General Review. Dep Food Eng Ege Univ Izmir. 2014;137:47-51.

3. ${ }^{\star \star \star}$ European Medicines Agency, Committee for Human Medicinal Products - Guideline on the Investigation of Drug Interactions, 2013.

4. Hawkey C, Kahan A, Steinbruck K, et al. - Gastrointestinal tolerability of meloxicam compared to diclofenac in osteoarthritis patients. $\mathrm{Br} J$ Rheumatol. 1998;37:937-945.

5. Gan TJ. - Diclofenac: an update on its mechanism of action and safety profile. Curr Med Res Opin. 2010;26:1715-1731.

6. Davies NM, Anderson KE. - Clinical Pharmacokinetics of Diclofenac. Clin Pharmacokinet. 1997;33:1-20.

7. Hinz B, Chevts J, Renner B, et al. - Bioavailability of diclofenac potassium at low doses. Br J Clin Pharmacol. 2005;59:80-84.

8. Walter K, von Nieciecki A. - Relative bioavailability of diclofenac after a single administration of a new multiple-unit formulation with entericcoated pellets. Arzneimittel-Forschung-Drug Res. 2001;51:643-650.

9. Terhaag B, Gramatte T, Hrdlcka P, Richter K, Feller K. The influence of food on the absorption of diclofenac as a pure substance. Int $\mathrm{J}$ Clin Pharmacol Ther Toxicol. 1991;29:418-421.

10. Willis J, Kendall M, Jack D. - The influence of food on the absorption of diclofenac after single and multiple oral doses. Eur J Clin Pharmacol. 1981;19:33-37.

11. Ahmad AM. - Recent advances in pharmacokinetic modeling. Biopharm Drug Dispos. 2007;28:135-143.

12. ${ }^{\star \star \star}$ Pharmacology C. Center for Drug Evaluation - Clinical Pharmacology and Biopharmaceutics Review (S). 2009:1-5. 